\title{
Stage II Major Salivary Gland Cancer AJCC v8
}

National Cancer Institute

\section{Source}

National Cancer Institute. Stage II Major Salivary Gland Cancer A/CC v8. NCI Thesaurus. Code $C 132785$.

Stage II includes: T2, NO, MO. T2: Tumor measuring more than $2 \mathrm{~cm}$, but not more than $4 \mathrm{~cm}$ in greatest dimension without extraparenchymal extension. N0: No regional lymph node metastasis. M0: No distant metastasis. (AJCC 8th ed.) 